\title{
Les troubles de l'oralité alimentaire dans les maladies neuromusculaires
}

\section{Analyse et prise en charge}

\author{
Danielle Ginisty, Hélène Rak-Merkin, Abdelkhaled Kaddour-Brahim
}

Les difficultés de passage à une alimentation en morceaux sont d'une extrême fréquence chez les enfants atteints de pathologies neuromusculaires, en rapport direct avec le déficit moteur dans son expression bucco-faciale.

L'oralité alimentaire de l'enfant évolue avec les deux dentitions, de la tétée quand le bébé n'a pas encore de dents vers l'alimentation à la cuillère puis, avec l'éruption des dents lactéales, l'alimentation en morceaux débute.

Les difficultés de passage de l'alimentation liquide à une alimentation plus épaisse puis solide sont d'une extrême fréquence chez les enfants atteints de pathologies neuromusculaires [1, 2]).

\section{Premier temps de la déglutition}

Le premier temps de la déglutition correspond au conditionnement dans la cavité buccale du bol alimentaire avant la déglutition proprement dite qui est un temps réflexe.

Ce temps réflexe postérieur intervient dès que le bol alimentaire passe de la cavité buccale vers le pharynx au niveau du rideau vélaire.

Passer de la tétée du lait à la mastication d'aliments solides, en morceaux, se fait sur une période longue fonction de l'éruption des molaires et surtout des dernières qui sont indispensables (de 18 à 30 mois) avec un nouveau geste mandibulaire de mastication. - Lors de la tétée, la mandibule suit des mouvements de bielle avant-arrière avec une excursion sensiblement parallèle de la mandibule et de la langue qui vient au contact des lèvres et de la tétine ou du sein (déglutition de type I).

Professeur

des Universités, Paris Descartes, ancien Chef de Service chirurgie maxillo-faciale et Stomatologie, $\mathrm{CHU}$

Necker-Enfants Malade Hélène Rak-Merkin

Ancien Praticien Attaché Consultante, $\mathrm{CHU}$

Necker-Enfants Malades Abdelkhaled

Kaddour-Brahim

Praticien Hospitalier

CHU Necker-Enfants

Malades

Contact

danielle.ginisty@aphp.fr
- Mastiquer demande de connaitre le croquer puis de pouvoir triturer les morceaux avec une excursion latérale de la mandibule permettant l'écrasement des morceaux entre les molaires [3]. La déglutition se fait avec la langue en position de recul au palais (déglutition adulte).

\section{Éléments pouvant perturber cette évolution praxique}

Quels sont les éléments à prendre en compte pouvant perturber cette évolution praxique en cas de pathologies neuromusculaires?
- Bien manger, c'est d'abord manger sans douleurs

Des dents saines c'est bien le premier objectif préalable à toute amélioration de la qualité de l'alimentation [4].

En l'absence d'hygiène buccale régulière, les gingivites sont d'une extrême fréquence, majorées par la respiration buccale a fortiori en cas de ventilation sous pression positive ; les gingivites sont douloureuses, s'accompagnent de saignements des gencives au brossage; si le brossage n'est pas fait, les saignements comme le risque de caries augmentent. Les caries sont douloureuses à l'alimentation et sont à l'origine de foyers infectieux. Les cellulites et les phlegmons en sont la complication locorégionale la plus redoutable. Elles surviennent le plus souvent après des abcès dentaires à répétition.

La destruction des couronnes dentaires entraîne une réduction des possibilités masticatrices et peut majorer les difficultés alimentaires avec une stagnation pondérale.

Les soins dentaires sont donc le premier geste salvateur, si possible à l'état vigile, d'où l'importance du premier contact avec un praticien formé à l'abord de l'enfant, si possible au sein d'une équipe pluridisciplinaire, avec possibilité de relaxation psychomotrice ou équivalent.

L'anesthésie générale en cas de caries multiples ou compliquées d'abcès est souvent inévitable; elle permet des soins efficaces puisque tous les soins conservateurs et les extractions sont réalisés dans le même temps après radiographies si besoin.

Insistons sur le nécessaire travail préventif relayé en milieu éducatif.

La limitation du grignotage, l'arrêt du biberon lacté ou sucré et la réalisation d'un brossage efficient le soir au coucher en sont les principaux éléments.

Leur application dès l'éruption des dents lactéales est la première mesure à prendre. 
- Les contacts dentaires insuffisants

Meilleurs sont les contacts entre les arcades, meilleure est la mastication.

Les anomalies de croissance des maxillaires et particulièrement les béances (absence de contact entre les arcades dentaires) réduisent les contacts et l'efficacité de mastication (Figure 1).

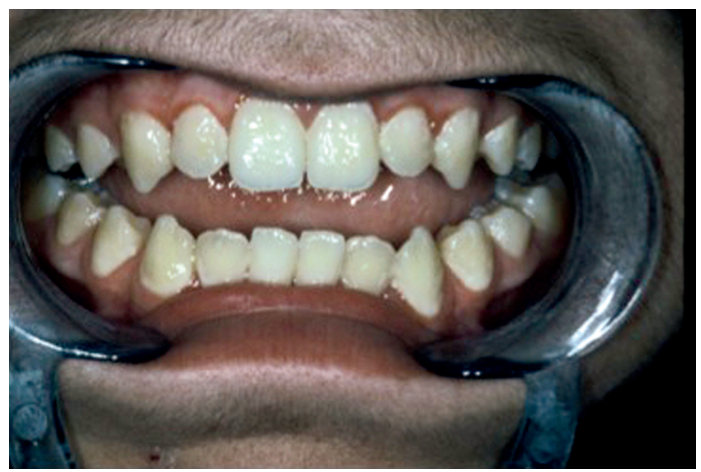

Figure 1

Béance antérieure.

En cas de MNM, plusieurs facteurs expliquent les difficultés de prise en charge, fonction de la gravité de la pathologie:

Les traitements orthopédiques

et particulièrement les corsets avec appui mentonnier

Ces traitements réalisent un traitement orthopédique a contrario sur la mandibule avec limitation de l'ouverture buccale (même si on enlève la mentonnière aux repas), et posture de défense de l'enfant menton en retrait ou évitement vers l'avant selon ses possibilités posturales.

D'où une mandibule en rétroposition dans le premier cas ou en proglissement parfois asymétrique dans le second (Figure 2).

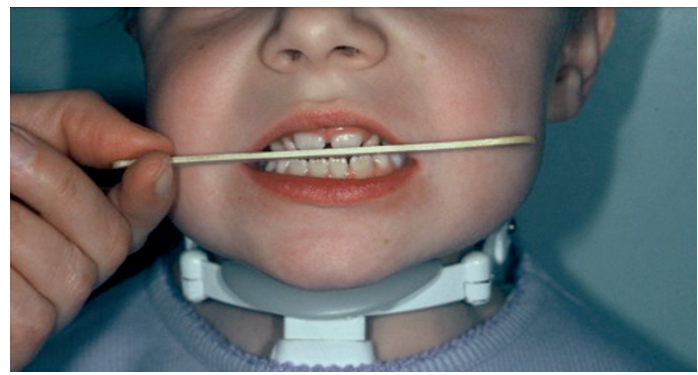

Figure 2

Promandibulie secondaire au port de la mentonnière d'un corset.

Aucun traitement orthodontique ne peut être efficace tant que le corset est en place.

Nous sommes donc très favorables aux nouvelles thérapeutiques orthopédiques micro-invasives qui limitent ce conflit corset-mandibule.

\section{Les difficultés ventilatoires}

Ces difficultés expliquent la position bouche ouverte avec une position basse de la langue et une promandibulie avec béance.

Les masques du commerce utilisés classiquement pour la VNI sont des forces extra-orales comparables à celles utilisées en orthopédie dento-faciale mais d'action opposée à la bonne croissance du massif facial (Figure 2).

L'utilisation d'un masque sur mesure limite ces effets. En cas d'indication de ventilation sur trachéotomie, la prise en charge orthodontique peut être plus aisée.

\section{Le déséquilibre musculaire entre langue,} muscles masticateurs et mimique faciale

Le déficit moteur des muscles masticateurs entraîne à la fois faiblesse de mastication, limitation de l'ouverture buccale et inocclusion labiale, ce qui majore l'impact prédominant de la langue sur le développement de la mandibule [5].

Son interposition entre les arcades est à l'origine des béances (Figure 1) et de l'excès de croissance de la mandibule avec un maxillaire supérieur trop étroit d'où des contacts molaires peu performants. L'examen complet de la cavité buccale permet de différencier les anomalies accessibles à des traitements courants (troubles d'articulation phonétique ou des praxies de déglutition) des macroglossies (Figure 3) liées le plus souvent dans les maladies neuromusculaires à une infiltration graisseuse des fibres musculaires.

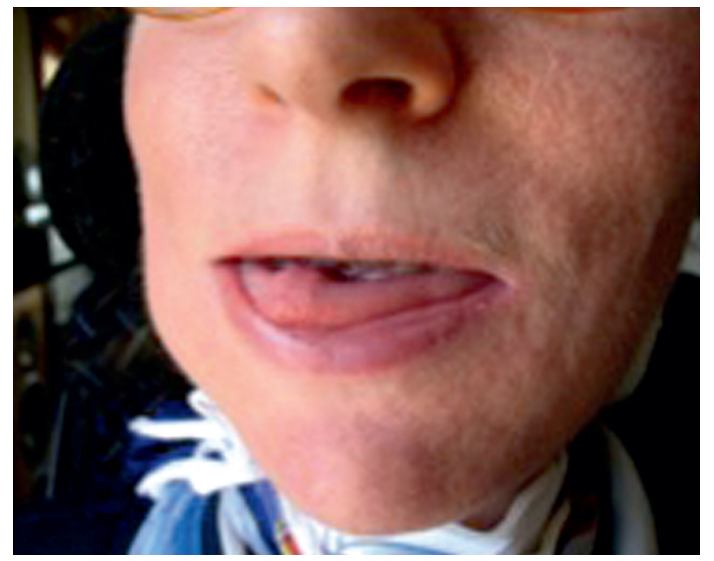

Figure 3

Macroglossie avec interposition permanente de la langue.

Les traitements orthodontiques après le début d'éruption des dents définitives permettent de favoriser la croissance du maxillaire supérieur à la condition d'une hygiène dentaire parfaite.

Mais ce traitement reste souvent insuffisant à long terme du fait de la macroglossie souvent d'apparition secondaire à l'adolescence. C'est pourquoi les glossoplasties sont dans notre expérience 
d'indication tardive, le plus souvent à la demande de l'adolescent, pour améliorer l'alimentation, l'articulation phonétique, tenter de limiter l'incontinence salivaire. Elles restent exceptionnelles dans notre expérience comme les ostéotomies correctrices car un tel programme orthodontico-chirurgical est lourd à mettre en place $[6,7]$.

Les dysfonctionnements des loges masticatrices Ces dysfonctionnements sont causés ou majorés par des contacts occlusaux anormaux sur des

dents saines ou traitées. Il s'agit d'anomalies fonctionnelles qui s'ajoutent aux troubles moteurs liés aux maladies neuromusculaires.

Outre les difficultés alimentaires, les signes d'appel sont un bruxisme (grincements dentaires), des crampes douloureuses des muscles masticateurs ou un trismus (limitation d'ouverture buccale) au réveil, aux repas, ou encore des morsures parfois répétées de la langue des lèvres ou de la muqueuse des joues. La méthode choisie d'occlusodontie appliquée à l'enfant consiste en des meulages sélectifs [8, 9] ; elle est indolore, respecte la hauteur des dents naturelles et nécessite une simple consultation, chaque séance étant réalisée à 3-5 semaines d'intervalle.

Les conditions pour réaliser ce traitement sont une denture stable, et précisément l'absence de traitement orthodontique. Elle procure une détente immédiate des muscles masticateurs et un engrènement dentaire instantané optimal.

Cette méthode a été évaluée chez 75 enfants (38 de sexe féminin) présentant une pathologie handicapante sans troubles du temps réflexe postérieur de la déglutition, dont neuf atteints de maladie neuromusculaire.

Le premier impact concerne le changement de texture alimentaire pour ceux qui mangeaient mixé (31 enfants), la durée et la fatigabilité lors des repas pour les autres.

L'amélioration a été constatée chez 64 enfants (85\%) après trois séances en moyenne (1 à 6).

- 21 enfants sur le groupe de 31 (68\%) ont acquis la mastication avec le passage à une alimentation en morceaux.

- Le gain de force de mastication est patent pour la quasi-totalité des enfants mangeant des morceaux avant l'occlusodontie (43 enfants sur 44) avec une diminution de la durée du repas pour trois, une normalisation des repas pour 11, une fatigabilité moindre pour 18 , une diversification alimentaire en particulier pour la viande (morceaux coupés moins finement) pour 10 enfants.

L'amélioration de la mastication n'a pas toujours été immédiate, le gain de force étant progressif jusqu'à 2 ans après le traitement (en denture stable et sans autre thérapeutique).
Le deuxième impact concerne la fréquence des fausses routes chez 12 enfants.

Les fausses routes occasionnelles en rapport avec la déglutition de morceaux non mastiqués ont totalement disparu dans sept cas et celles aux liquides dans neuf cas.

Enfin, un plus grand confort buccal a été obtenu - à défaut d'un changement alimentaire - chez onze enfants avec un impact positif sur bruxisme, trismus, morsures et fausses routes. Deux enfants de ce groupe présentaient une MNM (amyotrophie spinale de type II, et neuropathie sensitive sévère).

L'occlusodontie prend en compte des troubles fonctionnels d'origine occlusale favorisant un gain d'efficacité de mastication, de déglutition et un plus grand confort buccal.

\section{Conclusion}

L'examen stomatologique concerne l'ensemble des effecteurs du temps buccal avec un impact sur les praxies alimentaires, phonétiques ainsi que sur la croissance faciale.

L'analyse des anomalies permet de définir un plan de traitement spécifique tenant compte de la pathologie de chaque enfant et intégré au plan de traitement pédiatrique.

Feeding disorders in neuromuscular diseases: analysis and management

\section{LIENS D'INTÉRÊT}

Les auteurs déclarent n'avoir aucun lien d'intérêt concernant les données publiées dans cet article.

\section{RÉFÉRENCES}

1. Philpot J, Bagnall A, King C, et al. Feeding problems in merosin deficient congenital muscular dystrophy. Arch Dis Child 1999 ; 80 : 542-7.

2. Sparks S, Quijano-Roy S, Harper A, et al. Congenital muscular dystrophy overview. In : Pagon RA, Adam MP, Ardinger HH, et al., eds. GeneReviews $^{\circledR}$. Seattle (WA) University of Washington, 1993-2016.

3. Gola R, Chossegros C, Orthlieb JD. Syndrome algo-dysfonctionnel de l'appareil manducateur. Paris : Masson, 1992.

4. Law CS, Sheehan M, Needleman HL. Centronuclear myopathy and nursing pattern caries: management of a 1 year old. $J$ Clin Pediatr Dent 1995 ; 20 : 69-72.

5. Barker JH, David DJ Management of facial dysmorphogenesis in nemaline myopathy: a case report. World J Orthod 2005 ; 6 : 156-60.

6. Miller JR. Orthodontic treatment of a patient with Duchenne muscular dystrophy and macroglossia: how informed consent was critical to success. Am J Orthod Dentofacial Orthop 2013 ; 144 : 890-8

7. Bezak BJ, Arce KA, Jacob A, Van Ess J. Orthognathic surgery in patients with congenital myopathies and congenital muscular dystrophies: case series and review of the literature. J Oral Maxillofac Surg 2016 ; 74 : 601-9.

8. Rigolet DA. Signes et diagnostic des troubles de l'occlusion. Acta Odonto-Stomatol $1976 ; 114 ; 253-8$.

9. Rak-Merkin H, Ginisty D. Le bruxisme ou la partie émergée de l'iceberg : comment l'éliminer. Pediatr Prat 2007 ; 185 : 10-2. 